\title{
Local hydroxyl adsorption geometry on $\mathrm{TiO}_{2}(110)$
}

\author{
W. Unterberger, ${ }^{1}$ T. J. Lerotholi, ${ }^{2}$ E. A. Kröger, ${ }^{1}$ M. J. Knight, ${ }^{2}$ D. A. Duncan, ${ }^{2}$ D. Kreikemeyer-Lorenzo, ${ }^{1}$ K. A. Hogan, ${ }^{3}$ \\ D. C. Jackson, ${ }^{2}$ R. Włodarczyk, ${ }^{4}$ M. Sierka, ${ }^{4}$ J. Sauer, ${ }^{4}$ and D. P. Woodruff ${ }^{2, *}$ \\ ${ }^{1}$ Fritz-Haber Institut der MPG, Faradayweg 4-6, D14195 Berlin, Germany \\ ${ }^{2}$ Physics Department, University of Warwick, Coventry CV4 7AL, United Kingdom \\ ${ }^{3}$ Department of Chemical \& Biological Sciences, University of Huddersfield HD1 3DH, United Kingdom \\ ${ }^{4}$ Institute of Chemistry, Humboldt University Berlin, Unter den Linden 6, 10009 Berlin, Germany
}

(Received 20 June 2011; published 28 September 2011)

\begin{abstract}
The local structure of the hydroxyl species on the rutile $\mathrm{TiO}_{2}(110)$ surface has been determined both experimentally and computationally. The experimental study exploited chemical state-specific $\mathrm{O} 1 \mathrm{~s}$ scannedenergy mode photoelectron diffraction from a surface exposed to atomic hydrogen, while density functional theory calculations were used to provide complementary information. As expected on the basis of previous studies, the bridging $\mathrm{O}$ atoms of the clean surface are hydroxylated, but this causes surprisingly small changes in the surrounding surface relaxation. Experiment and theory are in good agreement regarding the magnitude of these atomic movements. Specifically, the Ti- $\mathrm{O}_{\mathrm{OH}}$ surface bond is significantly longer (by $0.10-0.15 \AA$ ) than that of Ti- $\mathrm{O}_{\text {bridging }}$ bonds on the clean surface.
\end{abstract}

DOI: 10.1103/PhysRevB.84.115461

PACS number(s): 68.43.Fg, 68.47.Gh, 68.43.Bc, 68.35.B-

\section{INTRODUCTION}

The (110) surface of the rutile phase of $\mathrm{TiO}_{2}$ is the most studied of all oxide surfaces, ${ }^{1,2}$ not only because of the technological importance of titania but also because it is relatively straightforward to prepare this surface in such a way that the substrate is conducting and thus accessible to electron spectroscopies and scattering experiments. $\mathrm{TiO}_{2}(110)$ is thus a model surface for the exploration of a range of phenomena in oxide surface chemistry. Understanding hydroxylated oxide surfaces is particularly important because in realistic environments (in air, as well as at aqueous interfaces), a high level of hydroxylation is likely to occur. The interaction of water with $\mathrm{TiO}_{2}(110)$ has been studied extensively, both experimentally and computationally ${ }^{1,3}$ and particularly in view of the discovery some 30 years ago of the photochemical production of hydrogen from water over titania. ${ }^{4}$ The clean $\mathrm{TiO}_{2}(110)$ surface is terminated by rows of oxygen atoms that bridge fully (sixfold) coordinated $\mathrm{Ti}$ atoms below and by (fivefold) undercoordinated surface $\mathrm{Ti}$ atoms (see Fig. 1, in which the atomic labeling of Ref. 1 has been used). While it seems to be established experimentally that, under standard (ultrahigh vacuum, or UHV) surface science conditions, water will not react with a perfect surface, such interaction does occur at the sites of bridging oxygen vacancies. At these sites, molecular water dissociates, leaving an $\mathrm{OH}$ species at the vacancy site, while the remaining $\mathrm{H}$ atoms hydroxylate an adjacent bridging $\mathrm{O}$ atom, leaving two bridging $\mathrm{OH}$ species. This basic process has been followed on an atomic scale by scanning tunneling microscopy (STM) $)^{5,6}$ and has been described in computational studies, 3,6 although some calculations also predict facile dissociation on the perfect surface (see Refs. 7 and 8 for a discussion of this literature).

Despite the many surface-science studies of $\mathrm{TiO}_{2}(110)$, there are few quantitative structure determinations, and most of these have been of the clean $(1 \times 1)$ surface; significant relaxations of the positions of the surface atoms occur relative to an ideal bulk termination, and after some early controversies over the magnitude and sign of the changes in the interlayer spacings, ${ }^{9-14}$ more recent investigations (both experimental ${ }^{10-12,14,15}$ and theoretical ${ }^{16-18}$ ) all seem to agree quite well. There are also two experimental studies of the local structure of hydroxyl species on $\mathrm{TiO}_{2}(110)$, both using scanned-energy mode photoelectron diffraction $(\mathrm{PhD})$ but both involving coadsorbed carboxylate species. The $\mathrm{PhD}$ technique $^{19,20}$ exploits the coherent interference of the directly emitted component of the photoelectron wavefield, from a core level of a surface atom, with components of the same wavefield elastically scattered by the surrounding atoms. If the photon energy, and thus the photoelectron energy, is scanned, these scattering paths switch in and out of phase with the directly emitted wavefield component due to the changing photoelectron wavelength. The resulting modulations in photoemission intensity with energy, at different emission directions, then provide a means of determining the emitter site relative to the surrounding scattering atoms through the use of multiple scattering simulations. The use of core level photoemission renders the technique element specific through the core-level binding energy, but chemical-state specificity is also achievable due to core level shifts (CLSs) associated with different bonding geometries of emitter atoms of the same element. In the case of oxygen-containing adsorbates on oxide surfaces, the chemical shift of the $\mathrm{O} 1$ s photoelectron binding energy is a key factor that allows the $\mathrm{PhD}$ technique to be used, distinguishing the emission from the absorbate $\mathrm{O}$ atoms and the emission from $\mathrm{O}$ atoms in the underlying oxide. In the case of interaction of the $\mathrm{TiO}_{2}(110)$ surface with formic acid ${ }^{21}$ and with glycine, ${ }^{22}$ the resulting coadsorbed formate (HCOO) and hydroxyl species and the glycinate $\left(\mathrm{NH}_{2} \mathrm{CH}_{2} \mathrm{COO}\right)$ and hydroxyl species, respectively, are found to have essentially the same $\mathrm{O}$ 1s CLS with respect to the oxidic O 1s emission. As a result, the $\mathrm{PhD}$ data recorded from the adsorbate $\mathrm{O} 1 \mathrm{~s}$ signal from these surfaces comprise an incoherent sum of the $\mathrm{PhD}$ from the hydroxyl $\mathrm{O}$ atoms and from the two carboxylate $\mathrm{O}$ atoms. Despite this complication, the experiments on these two systems clearly identified the hydroxyl species as being located in the expected bridging sites. 


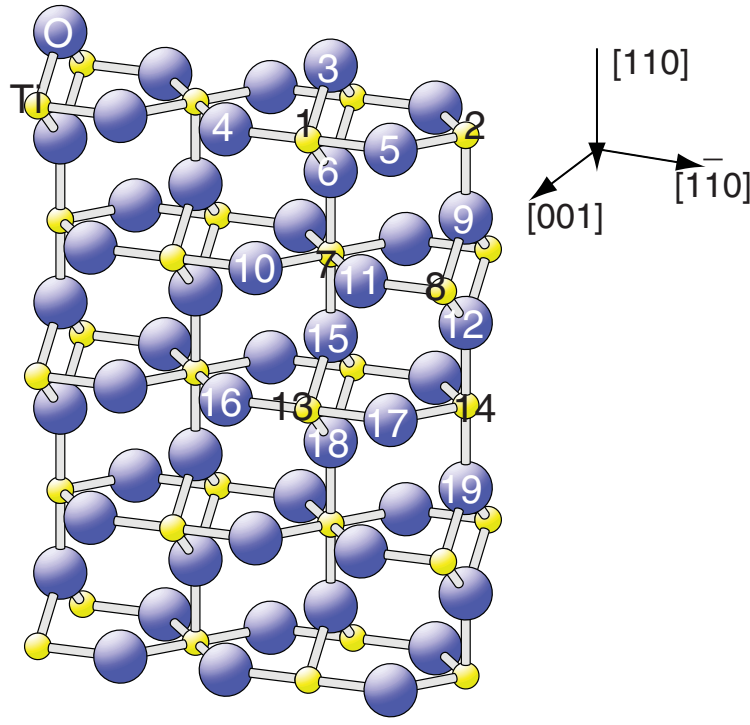

FIG. 1. (Color online) Schematic model of the clean $\mathrm{TiO}_{2}(110)$ surface with the $\mathrm{Ti}$ and $\mathrm{O}$ atoms of the different layers labeled following the convention of Ref. 1. These labels are used in identifying the displacements listed in Table I.

For the results presented here, we studied a $\mathrm{TiO}_{2}(110)$ surface with only adsorbed hydroxyl species. In principle, this could be studied experimentally by exploiting water dissociation at oxygen vacancy sites. However, starting from a well-prepared clean surface, the resulting surface concentration of $\mathrm{OH}$ sites is low (only a few percent), leading to a weak associated $\mathrm{O} 1 \mathrm{~s}$ photoemission shoulder on the oxidic $\mathrm{O}$ 1s peak. Artificially creating more surface defects prior to oxygen dosing using ion bombardment or electron-stimulated desorption offers a potential route to achieving a higher hydroxyl coverage, although it is then difficult to be sure that no other more extensive surface damage occurs. Instead, we have prepared our hydroxylated surface by exposure of the clean surface to atomic hydrogen, a method less likely to lead to larger-scale defects on the surface. Because it is not possible to dissociate water on a perfect $\mathrm{TiO}_{2}(110)$ surface (which would result in added $\mathrm{OH}$ and $\mathrm{H}$ species), the hydroxylated surface prepared either by water dissociation at $\mathrm{O}$ vacancies or by atomic $\mathrm{H}$ adsorption must lead to a surface that is formally chemically reduced. To complement our experimental structure determination, we also report the results of density functional theory (DFT) calculations, allowing us to explore some possible structural modifications and compare the measured and calculated surface relaxations of the hydroxylated surface.

\section{EXPERIMENTAL AND COMPUTATIONAL DETAILS}

The experiments were conducted in a conventional UHV surface science end station equipped with the usual facilities for sample cleaning, heating, and cooling. This instrument was installed on the UE56/2-PGM-1 beamline of BESSY II, which comprises a 56-mm-period undulator followed by a plane grating monochromator. ${ }^{23}$ Sample characterization in situ was achieved by low-energy electron diffraction
(LEED) and by soft x-ray photoelectron spectroscopy (SXPS) using the incident synchrotron radiation. These wide-scan SXPS spectra, and the narrow-scan $O$ 1s spectra used in the $\mathrm{PhD}$ measurements, were obtained using an Omicron EA-125HR 125-mm-mean-radius hemispherical electrostatic analyzer equipped with seven-channeltron parallel detection, which was mounted at a fixed angle of $60^{\circ}$ to the incident $x$ radiation in the same horizontal plane as that of the polarization vector of the radiation.

A clean, well-characterized rutile $\mathrm{TiO}_{2}(110)$ surface was prepared by briefly bombarding with $\mathrm{Ar}^{+}$ions at an energy of $500 \mathrm{eV}$, followed by annealing in UHV at approximately $830 \mathrm{~K}$. This treatment led to a sharp $(1 \times 1)$ LEED pattern and a Ti $2 p$ photoemission spectrum showing no significant high kinetic energy shoulder. Exposure to atomic $\mathrm{H}$ at a sample temperature of $140 \mathrm{~K}$ was achieved through the use of a commercial tube cracker (Oxford Applied Research), with a main chamber pressure of $\sim 2 \times 10^{-7} \mathrm{mbar}_{2}$, for 10 minutes. The treatment led to a significant enhancement of the intensity of the low kinetic energy shoulder on the $\mathrm{O} 1 \mathrm{~s}$ photoemission peak, with a corresponding CLS of $\sim 1.6 \mathrm{eV}$ (Fig. 2), that is attributed to emission from hydroxylated $\mathrm{O}$ atoms on the surface. Doubling this exposure led to no detectable increase in the intensity of this $\mathrm{O} 1 \mathrm{~s}$ photoemission peak shoulder, implying near-saturation coverage. The $\mathrm{OH}$ coverage was estimated from the relative intensities of the hydroxyl and oxidic $\mathrm{O} 1 \mathrm{~s}$ component peaks, using a value of the attenuation length in $\mathrm{TiO}_{2}$ at a kinetic energy of $95 \mathrm{eV}$ of $5.7 \AA$, as calculated using the National Institute of Standards and Technology's EffectiveAttenuation-Length Database computer program. ${ }^{24}$ Using this approach, a hydroxyl coverage of $0.4-0.5$ monolayer (ML) was obtained, where $1 \mathrm{ML}$ corresponds to the number of bridging $\mathrm{O}$ atoms on a perfect clean surface. This coverage is higher

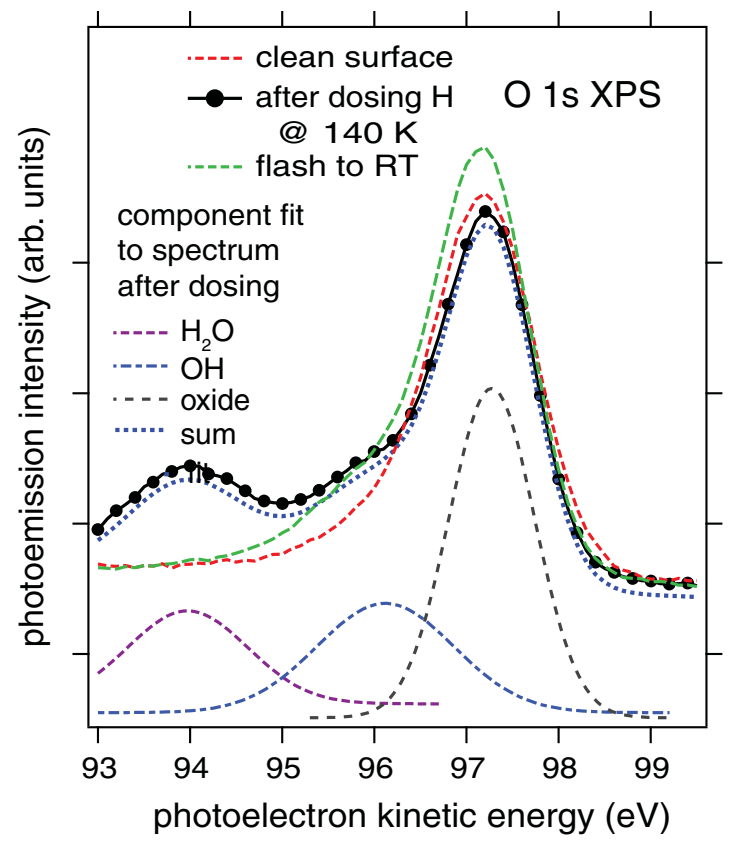

FIG. 2. (Color online) O 1s photoemission spectra recorded before and after $\mathrm{H}$ dosing, showing the two component $(\mathrm{OH}$ and oxidic) peaks. 
than the value of 0.23 ML estimated to correspond to saturation in an STM study ${ }^{25}$ but less that the 1 ML inferred from helium atom scattering experiments. ${ }^{26,27}$ Both of these earlier studies also used exposure to atomic hydrogen. No new ordered superstructure was seen using LEED in our experiments.

$\mathrm{O} 1 \mathrm{~s} \mathrm{PhD}$ data were obtained from this hydroxyl component by recording a sequence of photoelectron energy distribution curves (EDCs) around this photoemission peak, at equal steps in photon energy, in the photoelectron kinetic energy range of $\sim 60-320 \mathrm{eV}$. These data were measured at several polar emission angles in the range $0^{\circ}-60^{\circ}$ and in the two principal azimuths [001] and [1ㅜㅣ] (see Fig. 1). Data reduction followed our general PhD methodology (e.g., see Refs. 19 and 20), in which the individual $\mathrm{O} 1 \mathrm{~s}$ EDCs was fitted by the sum of two Gaussian peaks (hydroxyl and oxide), a step, and a template background. The integrated peak areas were then plotted as a function of photoelectron energy, and each final PhD modulation spectrum was obtained by subtraction of, and normalization by, a smooth spline function representing the nondiffractive intensity and instrumental factors.

DFT calculations were performed using the Vienna $a b$ initio simulation package, ${ }^{28,29}$ along with the Perdew, Burke, and Ernzerhof ${ }^{30,31}$ exchange-correlation functional. The electronion interactions were described by the projector augmented wave method, originally developed by Blöchl ${ }^{32}$ and adapted by Kresse and Joubert. ${ }^{33}$ Only the valence electrons were explicitly considered. Optimization of bulk rutile structure was carried out using a $1200-\mathrm{eV}$ cutoff for the plane wave basis set and a $(10 \times 10 \times 15)$ Monkhorst-Pack ${ }^{34}$ grid for the $k$-point sampling. The calculated lattice constants of $a_{0}=$ $4.663 \AA$ and $c_{0}=2.969 \AA$ are in good agreement with the experimental values of $a_{0}=4.594 \AA$ and $c_{0}=2.958 \AA .^{35}$

The $\mathrm{TiO}_{2}(110)$ surface was modeled using orthorhombic $(2 \times 1)$ and $(4 \times 1)$ slab supercells with lattice constants $a_{0}=5.938 \AA, b_{0}=6.595 \AA$ and $a_{0}=11.876 \AA, b_{0}=6.595 \AA$, respectively. The models contained five $\mathrm{TiO}_{2}$ layers, of which the lowermost three were fixed to their bulk positions. The lattice parameter $c_{0}$ was set to $35 \AA$, which resulted in a vacuum layer of approximately $20 \AA$ thickness, large enough to avoid slab interactions. Calculations for these models were performed with an energy cutoff of $400 \mathrm{eV}$ and $(3 \times 2 \times 1)$ and $(3 \times 5 \times 1)$ Monkhorst-Pack $k$-point meshes for the $(2 \times 1)$ and $(4 \times 1)$ structures, respectively.

Three models of the surface with 1-ML hydrogen coverage were constructed based on orthorhombic $(2 \times 1)$ supercell (unit cell composition: $\mathrm{Ti}_{20} \mathrm{O}_{40} \mathrm{H}_{2}$ ): (1) all hydrogen atoms bound to bridging $\mathrm{O}$ atoms (atom $\mathrm{O}$ in Fig. 1), with the $\mathrm{O}-\mathrm{H}$ bond perpendicular to the surface; (2) the same model but with alternately tilted $\mathrm{O}-\mathrm{H}$ bonds arranged in a zigzag manner; and (3) model 1 but with the $\mathrm{OH}$ species alternately offset in the [110] direction in a zigzag fashion. In addition, a model with 0.5 -ML hydrogen coverage was constructed in a $(4 \times 1)$ supercell by placing a hydrogen atom at every second bridging oxygen atom and allowing the $\mathrm{O}-\mathrm{H}$ bonds to tilt alternately in a zigzag fashion (unit cell composition: $\mathrm{Ti}_{40} \mathrm{O}_{80} \mathrm{H}_{2}$ ). The calculations for these hydrogenated surface models were performed using both spin-polarized and spinrestricted modes, which yield virtually identical structures (structural changes of $<0.01 \AA$ ).

\section{RESULTS}

The standard methodology for structure determination using PhD follows the trial-and-error approach common to most surface structural methods, in which the results of multiple-scattering simulations for a range of model structures are compared with the experimental modulation spectra and the model structure is modified to identify the best-fit structure. These calculations were performed with computer codes developed by Fritzsche ${ }^{36-38}$ that are based on the expansion of the final state wave function into a sum over all scattering pathways that the electron can take from the emitter atom to the detector outside the sample. For each model structure explored, a multiple scattering simulation of the selected experimental $\mathrm{PhD}$ spectra was performed, and the quality of agreement between theory and experiment was judged by the value of an objective reliability or $R$-factor, described more fully elsewhere. ${ }^{19,20}$ The variation of this $R$-factor value as a function of individual structural parameter values also provides a basis for estimating the precision of the ultimate best-fit structural solution. ${ }^{19,20}$ Typically, we find structures that are proved to be correct to give $R$ values less than $\sim 0.3$, although this value depends on the complexity of the structure, the amplitude of the $\mathrm{PhD}$ modulations, and the size of the experimental data set. For complex structures, often involving multiple sites, and for data sets that show only weak modulations, it is sometimes not possible to achieve a value lower than $\sim 0.4$. In the present case, the modulation amplitudes were weak (less than $\pm 10 \%$ ) in most emission directions; however, in the [001] azimuth at polar emission angles of $40^{\circ}-50^{\circ}$, somewhat stronger modulations (around $\pm 15 \%-20 \%$ ), with a single long dominant period, are seen. $\mathrm{PhD}$ modulation spectra of this type typically occur when the emission direction corresponds to approximately $180^{\circ}$ backscattering from a near-neighbor substrate atom, so this observation is entirely consistent with our expectation that the hydroxylated $\mathrm{O}$ atoms are at the bridging sites on the surface (see Fig. 1).

To perform the more rigorous and quantitative structure determination, through multiple-scattering simulations, we therefore focused on four $\mathrm{O} 1 \mathrm{~s}$ ( $\mathrm{OH}$ component) $\mathrm{PhD}$ spectra recorded in the [001] azimuth: those recorded at polar emission angles of $40^{\circ}, 50^{\circ}$, and $60^{\circ}$, which showed the largest modulations, together with that recorded in the high-symmetry normal emission direction. In addition, calculations were performed for emission in the [1 $\overline{10}$ ] azimuth at polar emission angles of $40^{\circ}$ and $50^{\circ}$; these spectra show very weak modulations and may therefore be expected to be challenging to reproduce in simulations, but simulations for a correct structure should, at the least, also show comparably weak modulations. $R$-factor values were calculated for both the set of four spectra showing the strongest modulations and the complete set of six spectra. The multiple scattering simulations performed included the effect of scattering from the $\mathrm{Ti}$ and $\mathrm{O}$ atoms of the substrate but not from the very weakly scattering $\mathrm{H}$ atoms. Initial calculations were conducted using a $(1 \times 1)$ structure with all bridging oxygen atoms as hydroxylated emitters, although this implies a higher (1 ML) hydroxyl coverage than was estimated in the experiments. Subsequently, the final calculations were conducted assuming an ordered $(2 \times 1) \mathrm{OH}$ structure, with alternate bridging oxygen atoms being hydroxylated in the 
bridging-site model. This same model formed the basis of the DFT calculations (because $\mathrm{PhD}$ is insensitive to the $\mathrm{H}$ atom locations, any tilting of $\mathrm{O}-\mathrm{H}$ bonds does not influence the $\mathrm{PhD}$ results). This periodicity corresponds closely to the $0.5-\mathrm{ML}$ $\mathrm{OH}$ coverage of the experiments, although no ordered structure was observed experimentally. We stress, however, that the $\mathrm{PhD}$ technique is essentially local in character, being sensitive primarily to the location of near-neighbor atoms relative to the emitter and specifically to substrate atoms that lie below the emitter atom. Any contribution of scattering from coplanar $\mathrm{OH}$ neighbors is minimal, so the assumed periodicity of the surface has no significant influence on the structural solution. However, the lower-coverage $(2 \times 1)$ periodicity does allow certain lateral substrate relaxations to occur (described later), which are symmetry forbidden in a $(1 \times 1)$ structure.

Initial model calculations focused on the basic anticipated structure, i.e., $\mathrm{OH}$ species in bridging sites on the surface, but assumed the structure of the underlying surface corresponds to termination of the ideal bulk, with no near-surface relaxations. In these calculations, only the height of the $\mathrm{O}$ atoms in the $\mathrm{OH}$ species, relative to the underlying surface, was varied. Comparison of the results of these simulations with the experimental spectra [Fig. 3(a)] shows that periodicity and phase of the main modulations are reproduced well, but the fine structure is rather poorly reproduced, as reflected in the overall $R$-factor values of 0.43 for four spectra and 0.41 for the full set of six spectra. Some exploration of the effects of limited surface relaxations was undertaken, with only marginal improvements in the agreement with experiment. However, two rather different modifications of the model were also explored. One of these is the possibility that a second site for the $\mathrm{OH}$ may be cooccupied, the most obvious possibility being atop the fivefold-coordinated Ti surface atoms. From (a)

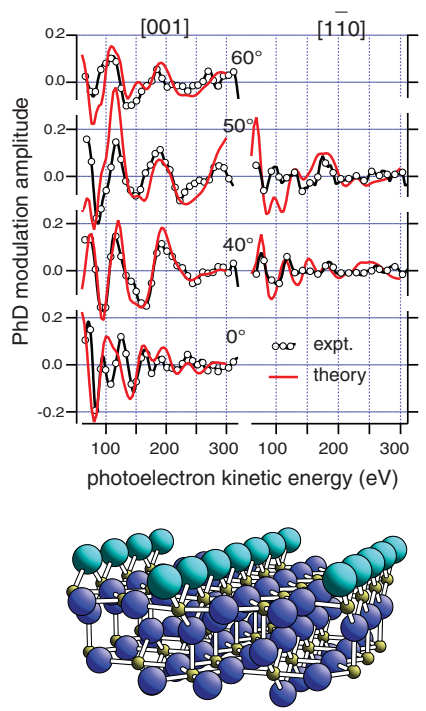

(b)

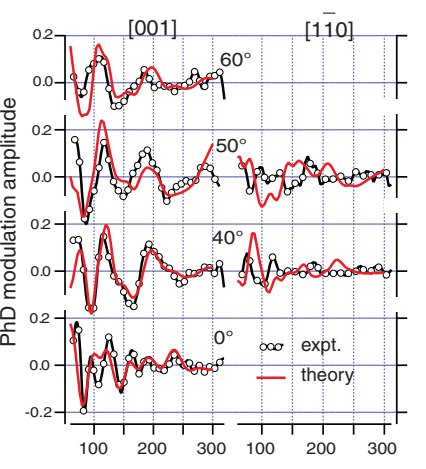

photoelectron kinetic energy $(\mathrm{eV})$

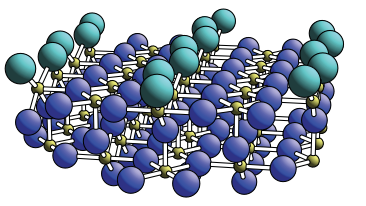

FIG. 3. (Color online) Comparison of the experimental $\mathrm{PhD}$ modulation spectra with the results of simulations for (a) the "simple" bridging-site model and (b) the "zigzag" $\mathrm{OH}$ row model, both on a bulk-terminated substrate. The $\mathrm{O}$ atoms of the $\mathrm{OH}$ species in the outermost layer are shown in a paler color. No $\mathrm{H}$ atoms are shown. the point of view of the $\mathrm{PhD}$ technique, this possibility is structurally similar to the subsurface $\mathrm{H}$ site proposed as a result of a recent combined STM/DFT investigation. ${ }^{39}$ Specifically, the subsurface $\mathrm{H}$ in this model lies adjacent to an $\mathrm{O}$ atom directly below a bridging $\mathrm{O}$ atom (atom 6 in Fig. 1), and this $\mathrm{O}$ atom lies atop an underlying $\mathrm{Ti}$ atom. From the point of view of the underlying substrate atoms, therefore, this site is equivalent to the adsorption site atop a fivefold-coordinated Ti surface atom. In this local atop geometry, normal emission corresponds to the favored $180^{\circ}$ near-neighbor backscattering geometry, so partial occupation of such a site may be expected to modify significantly the normal emission spectrum that is fitted poorly [Fig. 3(a)] by the model based only on bridging hydroxyl species on an unrelaxed substrate. Model calculations using this mixed-site model failed, however, to produce any significant improvement in the quality of the agreement between experiment and theory.

An alternative structural modification that was found to improve the agreement involved lateral displacements of the

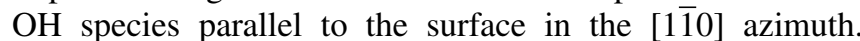
Specifically, in this model, the $\mathrm{OH}$ species (in this case, assumed to have a coverage of $1 \mathrm{ML}$ ) are alternately displaced in opposite directions within this azimuth to produce zigzag rows; as shown in Fig. 3(b), this model, with displacement amplitudes of $\sim 0.25 \AA$, led to a significantly lower value of the $R$-factor of 0.33 for the four-spectrum data set, although for the full six-spectrum data set the $R$-factor increased to 0.46 . Nevertheless, this structure clearly shows better agreement for the normal emission $\mathrm{PhD}$ spectrum. A possible rationale for such a distortion of the surface is the finding, in an investigation of the diffusion of hydrogen on $\mathrm{TiO}_{2}(110)$, of evidence for significant repulsive $\mathrm{OH}-\mathrm{OH}$ interactions. ${ }^{40}$ If there were to be high local $\mathrm{OH}$ coverage, these repulsive interactions could lead to compressive surface stress that may be relieved by a zigzag distortion. If the $\mathrm{OH}-\mathrm{OH}$ interactions are repulsive, we might expect that, at an average coverage of $\sim 0.5 \mathrm{ML}$, these nearest-neighbor $\mathrm{OH}$ sites would not be occupied. A further key question is whether such a distortion is energetically favorable, and it was for this purpose, initially, that the parallel DFT calculations were performed. The conclusion from these calculations is that this zigzag distortion is not stable. Energy minimization starting from such a structure led to the amplitude of the distortions falling to zero.

What the DFT results also revealed, however, was that the hydroxylated surface displays significant relaxations of the interlayer spacings of the near-surface region, relative to an ideally terminated bulk structure. The computed relaxations are quite similar to those of the clean surface. The effect of these relaxations on the $\mathrm{PhD}$ simulations was therefore determined by performing simulations that started from a structure using the exact DFT structural parameter values for the relaxations, but then allowing these to be reoptimized using a new automated fitting procedure, based on a "particle swarm optimization" algorithm. ${ }^{41}$ For this purpose, the initial structural parameters were taken from the DFT calculation based on the $(4 \times 1)$ structure with two $\mathrm{OH}$ species per surface unit mesh and thus a coverage of $0.5 \mathrm{ML}$. This model differs only from a simple hydroxylated $(2 \times 1)$ structure in allowing alternating $\mathrm{O}-\mathrm{H}$ bond tilt orientations along the close-packed 
[001] direction. The structural reoptimization using the $\mathrm{PhD}$ data was undertaken because, based on comparisons of exact interatomic bondlengths from DFT and $\mathrm{PhD}$ in previous studies of other systems, some modest differences are to be expected. It is also necessary for the $\mathrm{PhD}$ calculations to use the experimental bulk lattice parameters and not the slightly different values found in the DFT calculations. PhD simulations based on the exact DFT structure yielded a $R$-factor value of 0.51 (four spectra). However, this value was reduced to 0.23 for four spectra (and to 0.26 for six spectra) by optimization of the structural parameter values. The key conclusion is that incorporating a full set of near-surface relaxations leads to the best agreement between experiment and theory for the $\mathrm{PhD}$ data. Specifically, this optimized structure has a significantly lower value of the $R$-factor than either the simple or the zigzag bridge structures on an unrelaxed surface. Fig. 4 provides visual confirmation of the consequence of the lower $R$-factor, with agreement between theory and experiment now being generally good, even for the two very weakly modulated spectra recorded in the [1 $1 \overline{1} 0]$ azimuth.

It is interesting to compare the magnitude and sign of the surface relaxations found for this hydroxylated surface with those of the clean $(1 \times 1)$ surface, and these values are given in Table I. These relaxations have been investigated on the clean surface by LEED, medium energy ion scattering (MEIS), $\mathrm{PhD}$, and surface x-ray diffraction (SXRD). The values in Table I obtained from the quantitative LEED and SXRD studies are those reported in the reanalysis of the original experimental data, ${ }^{15}$ although these differ only slightly from those of the original reports. ${ }^{10,14}$ With one exception (described later), only relaxations perpendicular to the surface are reported here; a few small lateral relaxations of oxygen atoms found in some clean surface studies have not been explored in our study. The displacements are all given relative to the underlying (extended) bulk atomic positions, consistent with the reporting of all previous work. It is important to stress, however, that in a $\mathrm{PhD}$ study using an adsorbate emitter, the technique allows us to determine atomic positions relative to the emitter, not relative to the bulk. Moreover, as the electron scattering is essentially local in character, with the positions of near-neighbor atoms far more important than more distant atoms, any large relaxation in the deeper subsurface (to which we are not sensitive) would lead to systematic errors in the reported displacements of the nearsurface atoms when expressed relative to an extended bulk. However, as there is no evidence that such large subsurface movements occur (neither in the experimental studies of the clean surface nor in the DFT study of the hydroxylated surface), this effect is unlikely to be large. The essentially local character of the $\mathrm{PhD}$ technique means that the precision with which individual layers in the deeper surface can be located is poor, so no error estimates are given for the displacements of the lower layers; the PhD structural optimization calculations, started from the DFT minimum-energy structure, generally led to no movement of these deeper layers. By contrast, the results were relatively sensitive to the locations of the near-surface layers, as indicated in Table I, with the notable exception of the location of the fivefold-coordinated $\mathrm{Ti}$ atoms in the surface.
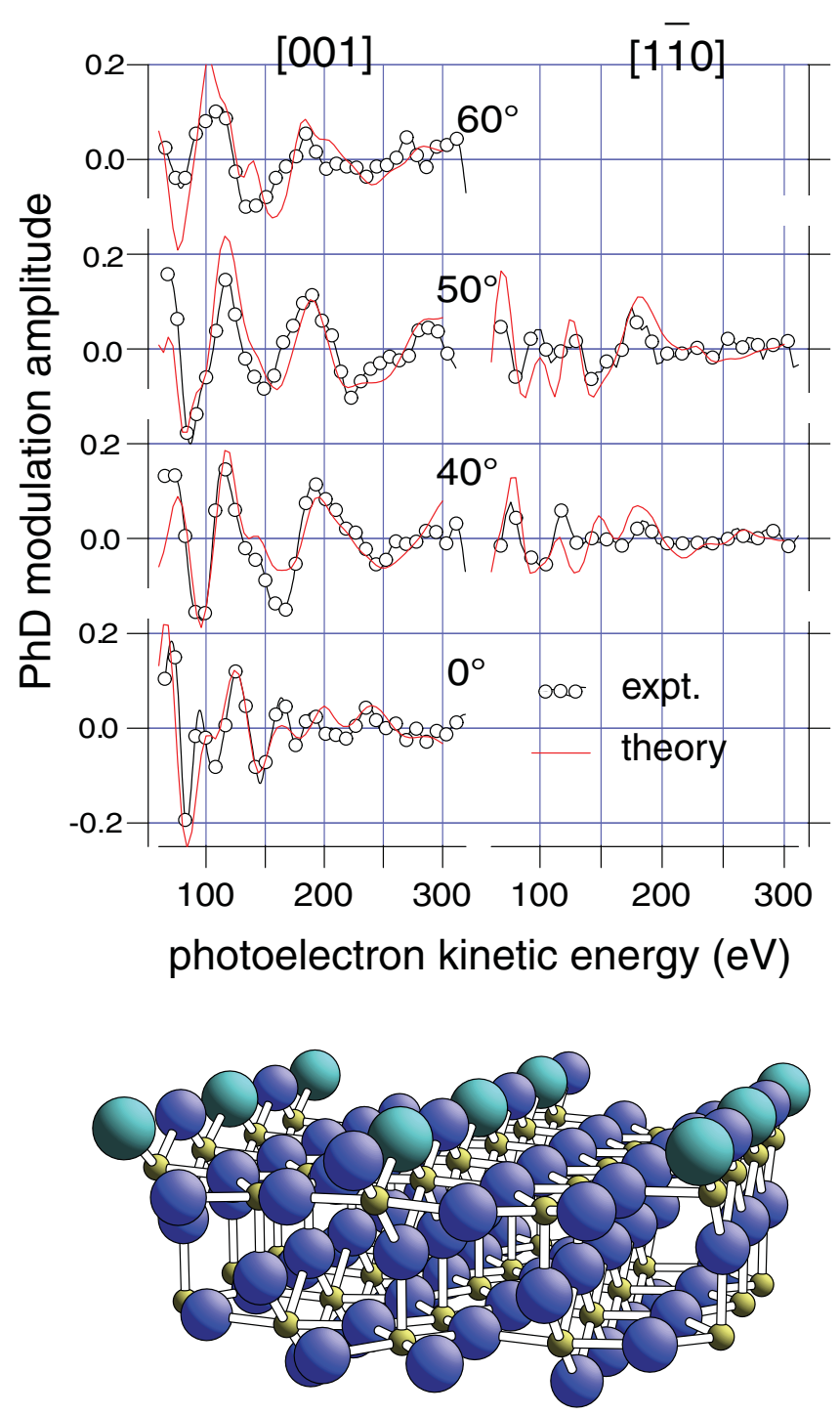

FIG. 4. (Color online) Comparison of the experimental PhD modulation spectra with the results of simulations for the relaxed bridging-site model based on reoptimization of the structural parameter values obtained in the DFT minimum-energy structure. The $\mathrm{O}$ atoms of the $\mathrm{OH}$ species in the outermost layer are shown in a paler color. No $\mathrm{H}$ atoms are shown.

One interesting result is that both the $\mathrm{PhD}$ and the DFT structures for the hydroxylated surface show values of the surface relaxations similar to those of the clean surface. On metal surfaces, which generally show a contraction of the outermost layer spacing in their clean state, adsorption commonly leads to this relaxation being greatly reduced, leaving a near-perfect bulk termination. Hydroxylation of the $\mathrm{TiO}_{2}(110)$ surface clearly does not have this effect, with all surface relaxations being, within the limits of the experimental precision, unchanged from those of the clean surface. The larger $(2 \times 1)$ surface mesh does allow some lateral displacements of atoms to occur that are symmetry forbidden on the $(1 \times 1)$ clean surface. Most notably, the spacing between the two sixfold-coordinated $\mathrm{Ti}$ atoms that are bridged by $\mathrm{OH}$ need not be the same as those bridged by the 
TABLE I. Displacements of the near-surface $\mathrm{Ti}$ and $\mathrm{O}$ atoms in $\mathrm{TiO}_{2}(110)$ relative to an ideal bulk-terminated structure for the clean surface (four experimental values) and the hydroxylated surface (the $\mathrm{PhD}$ and $\mathrm{DFT}$ results of the present work). $\Delta z$ values are displacements, in angstroms, defined as positive for an outward relaxation and are followed, in brackets, by the estimated error in units of $0.01 \AA$. $\Delta x(1)$ is the magnitude of the displacements of the sixfold Ti surface atoms along [001] assuming a $(2 \times 1)-\mathrm{OH}$ structure. A positive value indicates the separation of the $\mathrm{Ti}$ atoms bridging the $\mathrm{OH}$ is larger than that of $\mathrm{Ti}$ atoms bridging the unreacted $\mathrm{O}$ atoms. Asymmetric error estimates given in the PhD study of the clean surface (Ref. 12) are shown as approximate symmetric errors for simplicity. Entries that are dashed (-) indicate that a fixed value of 0.00 was assumed. The unassigned errors for the atoms deeper below the surface in the $\mathrm{PhD}+\mathrm{OH}$ column are discussed in the text.

\begin{tabular}{|c|c|c|c|c|c|c|c|}
\hline Atom & Parameter & LEED clean $^{10,15}$ & MEIS clean ${ }^{11}$ & $\mathrm{PhD}$ clean $^{12}$ & SXRD clean ${ }^{14,15}$ & $\mathrm{PhD}+\mathrm{OH}$ & $\mathrm{DFT}+\mathrm{OH}$ \\
\hline (1) Ti sixfold & $\Delta z(1)$ & $0.24(3)$ & $0.19(7)$ & $0.19(12)$ & $0.25(1)$ & $0.29(6)$ & 0.12 \\
\hline (1) Ti sixfold & $\Delta x(1)$ & - & - & - & - & $0.14(5)$ & 0.07 \\
\hline (2) Ti fivefold & $\Delta z(2)$ & $-0.19(3)$ & $-0.09(9)$ & $-0.26(8)$ & $-0.11(1)$ & $-0.17(27)$ & -0.12 \\
\hline (3) $\mathrm{O}$ bridging & $\Delta z(3)$ & $0.08(5)$ & $0.13(16)$ & $0.17(15)$ & $0.10(4)$ & $0.12(5)(\mathrm{OH})$ & $0.15(\mathrm{OH})$ \\
\hline (4),(5) O in-plane & $\Delta z(4,5)$ & $0.19(8)$ & 0.05 & $0.00(25)$ & $0.17(3)$ & $0.17(9)$ & 0.23 \\
\hline (6) $\mathrm{O}$ lower bridging & $\Delta z(6)$ & $0.07(10)$ & $0.10(13)$ & $0.15(15)$ & $0.07(4)$ & $0.12(12)$ & 0.08 \\
\hline (7) Ti second layer & $\Delta z(7)$ & $0.14(3)$ & $-0.06(6)$ & $0.15(18)$ & $0.19(1)$ & $0.14(11)$ & 0.05 \\
\hline (8) Ti second layer & $\Delta z(8)$ & $-0.11(4)$ & $-0.09(9)$ & $-0.21(25)$ & $-0.08(1)$ & 0.00 & -0.05 \\
\hline (9) O below Ti fivefold & $\Delta z(9)$ & $0.00(8)$ & - & $-0.03(8)$ & $0.00(3)$ & 0.13 & 0.06 \\
\hline (10),(11) O second layer & $\Delta z(10,11)$ & $0.07(12)$ & - & - & $0.04(3)$ & 0.11 & 0.06 \\
\hline (12) O second layer & $\Delta z(12)$ & $-0.01(14)$ & - & - & $0.01(22)$ & 0.02 & 0.04 \\
\hline (13) Ti third layer & $\Delta z(13)$ & $0.06(9)$ & $0.00(7)$ & - & $0.08(1)$ & 0.00 & - \\
\hline (14) Ti third layer & $\Delta z(14)$ & $-0.07(7)$ & $-0.02(8)$ & - & $-0.04(1)$ & 0.00 & - \\
\hline
\end{tabular}

unreacted $\mathrm{O}$ atoms; indeed, both the $\mathrm{PhD}$ and the DFT results are consistent with lateral movements of these Ti atoms along the close-packed [1힐 direction such that the spacing at the $\mathrm{OH}$ site is increased, as shown in Table I.

One important structural parameter, which can be extracted only indirectly from the displacements from the atomic positions of a bulk-terminated solid shown in Table I, is the local height of the $\mathrm{O}_{\mathrm{OH}}$ atom above the nearest-neighbor (sixfoldcoordinated) $\mathrm{Ti}$ atoms and thus the $\mathrm{Ti}-\mathrm{O}_{\mathrm{OH}}$ bondlength. In the bulk crystal, the equivalent Ti-O bridging bonds have a length of $1.95 \AA$ (with an associated interlayer spacing of $1.27 \AA$ ). On the clean surface, the LEED and SXRD studies agree closely in the displacements of the relevant $\mathrm{Ti}$ and $\mathrm{O}$ atoms, leading to the length of the Ti-O bridging bond being reduced to $1.85 \pm 0.03 \AA$. The MEIS and PhD studies of the clean surface yield values much closer to those of the bulk of $1.93 \pm 0.11 \AA$, but the estimated precision is much lower and thus the difference from the LEED and SXRD values is not significant. Because the bridging $\mathrm{O}$ atoms are undercoordinated, the implied shortening of the Ti-O bond relative to its value in the bulk is not surprising. For the bridging $\mathrm{OH}$ species, the $\mathrm{Ti}-\mathrm{O}_{\mathrm{OH}}$ bondlengths is given in the DFT structure reported here as $2.04 \AA$, while the experimental $\mathrm{PhD}$ study yields a value of $1.97 \pm 0.05 \AA$. The implication is thus, as we might expect, that hydroxylation of the bridging $\mathrm{O}$ atoms leads to a significant increase in the relevant Ti-O bondlength relative to that of the clean surface. The DFT calculation gives the Ti-O distance for the unhydroxylated bridging $\mathrm{O}$ atom as $1.86 \AA$, unchanged from the clean surface; the $\mathrm{PhD}$ data are completely insensitive to the location of these $\mathrm{O}$ atoms that are approximately coplanar with the $\mathrm{O}(\mathrm{OH})$ emitter atoms. The DFT and $\mathrm{PhD}$ studies differ in the chemisorption bondlength and indeed the DFT value is larger, which are consistent with some other molecular adsorption systems, such analine on $\mathrm{Cu}(110),{ }^{42}$ although we have previously remarked ${ }^{21}$ that such calculations provide an excellent quantitative description of the geometry of the formate species on $\mathrm{TiO}_{2}(110)$. However, the difference between theory and experiment in the present case of $0.07 \pm 0.05 \AA$ is marginally significant. The precision in the $\mathrm{Ti}-\mathrm{O}_{\mathrm{OH}}$ bondlength in our $\mathrm{PhD}$ study is the best of any parameter and reflects that the technique is most sensitive to this nearest-neighbor emitter-scatterer distance. This reasonably good precision for the $\mathrm{Ti}-\mathrm{O}_{\mathrm{OH}}$ bondlength contrasts with that achieved for the surface Ti-O bondlengths in the PhD study of the clean surface, in which the $\mathrm{O} 1 \mathrm{~s}$ and Ti $2 p$ emission signals used in the analysis arise from many different surface and near-surface layers, greatly reducing the sensitivity to the local geometry of the emitter atoms in any one specific layer.

In the introduction, we remarked that two previous $\mathrm{PhD}$ studies have determined the $\mathrm{OH}$ adsorption geometry on $\mathrm{TiO}_{2}$ (110) but only when coadsorbed with either a formate or a glycinate species. Nevertheless, it is interesting to compare the measured $\mathrm{Ti}-\mathrm{O}_{\mathrm{OH}}$ bondlength values. With coadsorbed formate, the value was found to be $2.02 \pm 0.05 \AA,^{21}$ similar to the value of $2.01 \pm 0.03 \AA$ found with coadsorbed glycinate. ${ }^{22}$ Our new results suggest that in the absence of these coadsorbed carboxylate species, the $\mathrm{Ti}-\mathrm{O}_{\mathrm{OH}}$ bondlength may be slightly shorter, but the differences are not formally significant

\section{CONCLUSIONS}

The main results of an experimental $\mathrm{PhD}$ study of the local structure of $\mathrm{OH}$ species on $\mathrm{TiO}_{2}(110)$ agree well with those of parallel DFT calculations. As has been widely anticipated, it is the surface bridging $\mathrm{O}$ atoms that are hydroxylated, but the results also provide quantitative structural information. Specifically, the Ti- $\mathrm{O}_{\mathrm{OH}}$ surface bond is significantly longer (by 
$0.10-0.15 \AA$ ) than that of $\mathrm{Ti}-\mathrm{O}_{\text {bridging }}$ bonds on the clean surface. This change in local bonding and associated bondlength is not, however, accompanied by any significant changes in the relaxations of the surrounding surface atoms, which retain displacements, relative to an ideally bulk-terminated solid, essentially identical to those of the clean surface.

\section{ACKNOWLEDGMENTS}

The authors acknowledge the financial support of the Engineering and Physical Sciences Research Council (UK), and of the Deutsche Forschungsgemeinschaft through the Sonderforschungsbereich 546, together with the award of beamtime by the BESSY synchrotron radiation facility. *d.p.woodruff@warwick.ac.uk

${ }^{1}$ U. Diebold, Surf. Sci. Rep. 48, 53 (2003).

${ }^{2}$ C. L. Pang, R. Lindsay, and G. Thornton, Chem. Soc. Rev. 37, 2328 (2008).

${ }^{3}$ B. Hammer, S. Wendt, and F. Besenbacher, Top. Catal. 53, 423 (2010).

${ }^{4}$ A. Fujishima and K. Honda, Nature 238, 37 (1972).

${ }^{5}$ I. M. Brookes, C. A. Muryn, and G. Thornton, Phys. Rev. Lett. 87, 266103 (2001).

${ }^{6}$ R. Schaub, P. Thostrup, N. Lopez, E. Lægsgaard, I. Stensgaard, J. K. Nørskov, and F. Besenbacher, Phys. Rev. Lett. 87, 266104 (2001).

${ }^{7}$ F. Allegretti, S. O'Brien, M. Polcik, D. I. Sayago, and D. P. Woodruff, Phys. Rev. Lett, 95, 226104 (2005).

${ }^{8}$ F. Allegretti, S. O'Brien, M. Polcik, D. I. Sayago, and D. P. Woodruff, Surf. Sci. 600, 1487 (2006).

${ }^{9}$ G. Charlton, P. B. Howes, C. L. Nicklin, P. Steadman, J. S. G. Taylor, C. A. Muryn, S. P. Harte, J. Mercer, R. McGrath, D. Norman, T. S. Turner, and G. Thornton, Phys. Rev. Lett. 78, 495 (1997).

${ }^{10}$ R. Lindsay, A. Wander, A. Ernst, B. Montanari, G. Thornton, and N. M. Harrison, Phys. Rev. Lett. 94, 246102 (2005).

${ }^{11}$ G. S. Parkinson, M. A. Muñoz-Márquez, P. D. Quinn, M. J. Gladys, R. E. Tanner, D. P. Woodruff, P. Bailey, and T. C. Q. Noakes, Phys. Rev. B 73, 245409 (2006).

${ }^{12}$ E. A. Kröger, D. I. Sayago, F. Allegretti, M. J. Knight, M. Polcik, W. Unterberger, T. J. Lerotholi, K. A. Hogan, C. L. A. Lamont, and D. P. Woodruff, Phys. Rev. B 75, 195413 (2007).

${ }^{13}$ S. J. Thompson and S. P. Lewis, Phys. Rev. B 73, 073403 (2006).

${ }^{14}$ G. Cabailh, X. Torrelles, R. Lindsay, O. Bikondoa, I. Joumard, J. Zegenhagen, and G. Thornton, Phys. Rev. B 75, 241403 (2007).

${ }^{15}$ W. Busayaporn, X. Torrelles, A. Wander, S. Tomić, A. Ernst, B. Montanari, N. M. Harrison, O. Bikondoa, I. Joumard, J. Zegenhagen, G. Cabailh, G. Thornton, and R. Lindsay, Phys. Rev. B 81, 153404 (2010).

${ }^{16}$ V. Swamy, J. Muscat, J. D. Gale, and N. M. Harrison, Surf. Sci. 504, 115 (2002).

${ }^{17}$ K. J. Hameeuw, G. Cantele, D. Ninno, F. Trani, and G. Iadonisi, J. Chem. Phys. 124, 024708 (2006).

${ }^{18}$ F. Labat, P. Baranek, and C. Adamo, J. Chem. Theor. Comput. 4, 341 (2008).
${ }^{19}$ D. P. Woodruff and A. M. Bradshaw, Rep. Prog. Phys. 57, 1029 (1994).

${ }^{20}$ D. P. Woodruff, Surf. Sci. Rep. 62, 1 (2007).

${ }^{21}$ D. I. Sayago, M. Polcik, R. Lindsay, J. T. Hoeft, M. Kittel, R. L. Toomes, and D. P. Woodruff, J. Phys. Chem. B 108, 14316 (2004).

${ }^{22}$ T. J. Lerotholi, E. A. Kröger, M. J. Knight, W. Unterberger, K. Hogan, D. C. Jackson, C. L. A. Lamont, and D. P. Woodruff, Surf. Sci. 603, 2305 (2009).

${ }^{23}$ K. J. S. Sawhney, F. Senf, M. Scheer, F. Schäfers, J. Bahrdt, A. Gaupp, and W. Gudat, Nucl. Instrum. Meth. A 390, 395 (1997).

${ }^{24}$ C. J. Powell and A. Jablonski, NIST Electron Effective-AttenuationLength Database, version 1.1 (National Institute of Standards and Technology, Gaithersburg, MD, 2003).

${ }^{25}$ S. Suzuki, K. I. Fukui, H. Onishi, and Y. Iwasawa, Phys. Rev. Lett. 84, 2156 (2000).

${ }^{26}$ M. Kunat, U. Burghaus, and Ch. Wöll, Phys Chem Chem Phys 6 , 4203 (2004).

${ }^{27}$ Ch. Wöll, J. Phys. Condens. Matter 16, S2981 (2004).

${ }^{28}$ G. Kresse and J. Furthmüller, Comput. Mater. Sci. 6, 15 (1996).

${ }^{29}$ G. Kresse and J. Furthmüller, Phys. Rev. B 54, 11169 (1996).

${ }^{30}$ J. P. Perdew, K. Burke, and M. Ernzerhof, Phys. Rev. Lett. 77, 3865 (1996).

${ }^{31}$ J. P. Perdew, K. Burke, and M. Ernzerhof, Phys. Rev. Lett. 78, 1396 (1997).

${ }^{32}$ P. E. Blöchl, Phys. Rev. B 50, 17953 (1994).

${ }^{33}$ G. Kresse and D. Joubert, Phys. Rev. B 59, 1758 (1999).

${ }^{34}$ H. J. Monkhorst and J. D. Pack, Phys. Rev. B 13, 5188 (1976).

${ }^{35}$ R. A. Robie, P. M. Bethke, and K. M. Beardsley, US Geol. Surv. Bull. 1248 (1967).

${ }^{36}$ V. Fritzsche, J. Phys. Condens. Matter 2, 1413 (1990).

${ }^{37}$ V. Fritzsche, Surf. Sci. 265, 187 (1992).

${ }^{38}$ V. Fritzsche, Surf. Sci. 213, 648 (1989).

${ }^{39}$ G. H. Enevoldsen, H. P. Pinto, A. S. Foster, M. C. R. Jensen, W. A. Hofer, B. Hammer, J. V. Lauritsen, and F. Besenbacher, Phys. Rev. Lett. 102, 136103 (2009).

${ }^{40}$ S.-C. Li, Z. Zhang, D. Sheppard, B. D. Kay, J. M. White, Y. Du, I. Lyubinetsky, G. Henkelman, and Z. Dohnálek, J. Am. Chem. Soc. 130, 9080 (2008).

${ }^{41}$ D. A. Duncan, J. Choi, and D. P. Woodruff (submitted for publication).

${ }^{42}$ D. I. Sayago, M. Polcik, G. Nisbet, C. L. A. Lamont, and D. P. Woodruff, Surf. Sci. 590, 76 (2005). 